Enferm Bras 2020;19(5):372-80

https://doi.org/10.33233/eb.v19i5.3672

\title{
ARTIGO ORIGINAL \\ Avaliação da adesão à hemodiálise pelo doente renal crônico
}

Lessaiane Catiuscia Silva de Oliveira*, Gerlene Grudka Lira, M.Sc.**, Flávia Emília Cavalcante Valença Fernandes, D.Sc. ${ }^{* \star}$, Daiane de Ornelas ${ }^{*}$, Rachel Mola de Mattos, D.Sc. ${ }^{* \star *}$

${ }^{*}$ Enfermeira Nefrologista, Clínica de Nefrologia de Juazeiro (Clinefro), ${ }^{* *}$ Professora Assistente do Colegiado de Enfermagem da Universidade de Pernambuco Campus Petrolina, ${ }^{* * *}$ Professora Adjunta do Colegiado de Enfermagem da Universidade de Pernambuco Campus Petrolina, Clinefro, ${ }^{* * * * P r o f e s s o r a ~ A d j u n t a ~ d o ~ C o l e g i a d o ~ d e ~ E n f e r m a g e m ~ d a ~ U n i v e r s i d a d e ~ d e ~ P e r n a m b u c o ~}$ Campus Petrolina

Recebido em 26 de dezembro de 2019; aceito em 15 de outubro de 2020.

Correspondência: Flávia Emília Cavalcante Valença Fernandes, Av. Cardoso de Sá, s/n. Campus Universitário, Vila Eduardo, 56328-900 Petrolina PE

Lessaiane Catiuscia Silva de Oliveira: lessaiane_enf@hotmail.com

Gerlene Grudka Lira: gerlene.grudka@upe.br

Flávia Emília Cavalcante Valença Fernandes: flavia.fernandes@upe.br

Daiane de Ornelas: daianeornelas19964@hotmail.com

Rachel Mola de Mattos: rachel.mola@upe.br

\section{Resumo}

Introdução: A doença renal crônica vem crescendo mundialmente, podendo estar associada ao aumento da hipertensão e diabetes. Entre as modalidades de tratamento para esta doença está a hemodiálise, que necessita de adequada adesão terapêutica pelos pacientes para garantia de suas vidas. Objetivo: Avaliar o perfil sociodemográfico, clínico e a adesão a hemodiálise dos pacientes renais crônicos submetidos à hemodiálise em uma clínica de referência localizada no interior da Bahia, Brasil. Métodos: Trata-se de um estudo descritivo com abordagem quantitativa do qual participaram 174 pacientes renais crônicos. A coleta de dados aconteceu no período de outubro 2017 a fevereiro de 2018, através de um questionário de avaliação sobre a adesão do portador de doença renal crônica em hemodiálise, validado e adaptado no Brasil no ano de 2017. Resultados: A maioria dos participantes era do sexo masculino, casado, possuindo escolaridade e renda baixas. A hipertensão arterial foi a patologia que mais acometeu esses indivíduos, e a maioria demonstrou ser aderente ao tratamento. Existe um comprometimento dos profissionais de saúde, refletindo na qualidade do serviço prestado. Conclusão: Avaliar a adesão ao tratamento constitui uma ferramenta útil para aprimorar a prática gerencial e assistencial do serviço de nefrologia.

Palavras-chave: insuficiência renal crônica, cooperação do paciente, diálise renal, terapêutica, avaliação de resultado de intervenções terapêuticas.

\section{Abstract \\ Evaluation of hemodialysis adherence by chronic renal patients}

Introduction: Chronic kidney disease has been growing worldwide and may be associated with increased hypertension and diabetes. Among the treatment modalities for this disease is hemodialysis, which requires adequate therapeutic adherence by patients to guarantee their lives. Objective: To evaluate the sociodemographic, clinical profile and adherence to hemodialysis of chronic renal patients undergoing hemodialysis in a reference clinic located in the interior of Bahia, Brazil. Methods: This is a descriptive study with a quantitative approach conducted with 174 chronic renal patients. Data collection took place from October 2017 to February 2018, through an evaluation questionnaire on the adherence of patients with chronic kidney disease on hemodialysis, validated and adapted in Brazil in 2017. Results: Most of the participants were male, married, with low schooling and income. Hypertension was the pathology that most affected these individuals, and most have been shown to be adherent to treatment. There is a commitment of health professionals, reflecting on the quality of the service provided. Conclusion: Evaluating 
treatment adherence is a useful tool to improve the managerial and care practice of the nephrology service.

Keywords: chronic renal failure, patient cooperation, renal dialysis, therapy, evaluation of the result of therapeutic interventions.

\begin{abstract}
Resumen
Evaluación de la adherencia al tratamiento de hemodiálisis en pacientes renales crónicos Introducción: La enfermedad renal crónica ha estado creciendo en todo el mundo y puede estar asociada con el aumento de la hipertensión y la diabetes. Entre las modalidades de tratamiento para esta enfermedad se encuentra la hemodiálisis, que requiere una adecuada adherencia terapéutica por parte de los pacientes para garantizar su vida. Objetivo: Evaluar el perfil sociodemográfico, clínico y la adherencia al tratamiento de hemodiálisis por parte de pacientes renales crónicos sometidos a hemodiálisis en una clínica de referencia ubicada en el interior de Bahía, Brasil. Métodos: Este es un estudio descriptivo con un enfoque cuantitativo del cual participaron 174 pacientes renales crónicos. La recopilación de datos tuvo lugar de octubre de 2017 a febrero de 2018, a través de un cuestionario de evaluación sobre la adherencia de pacientes con enfermedad renal crónica en hemodiálisis, validado y adaptado en Brasil en 2017. Resultados: La mayoría de los participantes eran hombres, casados, con poca educación e ingresos bajos. La hipertensión fue la patología que más afectó a estos individuos, y la mayoría se ha demostrado que son adherentes al tratamiento. Existe un compromiso de los profesionales de la salud, reflexionando sobre la calidad del servicio prestado. Conclusión: Evaluar la adherencia al tratamiento es una herramienta útil para mejorar la práctica gerencial y de cuidado del servicio de nefrología.
\end{abstract}

Palabras-clave: insuficiencia renal crónica, cooperación del paciente, diálisis renal, terapia, evaluación del resultado de intervenciones terapéuticas.

Introdução

Dados epidemiológicos mostram que a doença renal crônica (DRC) ocorre em 5\% a 10\% da população mundial, no Brasil vêm aumentando gradativamente, fator que pode estar associado ao aumento de indivíduos diagnosticados com hipertensão e diabetes. O número de pacientes em programa dialítico no Brasil aumentou de 92.091 em 2010 para 112.004 em 2014 [1]. Dados do Inquérito Brasileiro de Diálise Crônica, em julho de 2016, apontou uma estimativa de 122.825 pacientes em diálise. As taxas nacionais de prevalência e incidência de pacientes em tratamento dialítico por milhão de habitantes (pmp), foram 596 (variação: 344 na região norte e 700 na região sudeste) e 193, respectivamente [2].

A DRC consiste em lesão e/ou perda lenta, progressiva e irreversível da função renal. Caracterizada por alterações da taxa de filtração glomerular, a DRC pode ser acompanhada ou não de lesão do parênquima renal, confirmada a partir de valores inferiores a $15 \mathrm{ml} / \mathrm{min} . / 1,73 \mathrm{~m}^{2}$, indicando o comprometimento renal grave e consequentemente uma terapia renal substitutiva [3].

Entre as modalidades de tratamento para estes pacientes estão a hemodiálise (HD), diálise peritoneal ou transplante renal, sendo a hemodiálise a terapia mais utilizada mundialmente [4]. A HD consiste no tratamento substitutivo da função renal realizada através de uma máquina que retira todo excesso de líquido e produtos metabólicos do corpo, quando os rins perdem sua capacidade. $O$ tratamento prescrito é feito numa média de três sessões semanais, com duração de três a cinco horas, dependendo da necessidade de cada paciente. Esta terapia é feita durante toda a vida até a realização do transplante renal [5].

A DRC representa um problema de saúde pública devido a sua alta complexidade de tratamento e elevados custos, considerando um aumento desses pacientes em regime de HD. Assim, os indivíduos devem zelar pela sua saúde, assumindo a responsabilidade, pelo seu bemestar e autocuidado [3].

O tratamento da HD traz repercussões para a vida do indivíduo, afetando sua qualidade de vida [6]. Por se tratar de uma experiência difícil e por vezes dolorosa, os pacientes devem adaptar-se as mudanças ocasionadas pelo tratamento, dentre as quais estão novos hábitos alimentares, rotina modificada, dependência familiar, e perda da autonomia [7].

Define-se a adesão terapêutica como grau de concordância entre o comportamento de uma pessoa referente à ingesta de medicamentos, realização de uma dieta, ou mudanças nos hábitos de vida, representando pontos importantes para o curso da enfermidade, evolução no tratamento e repercussões em sua qualidade de vida [8]. 
A não adesão terapêutica tem como consequências problemas no contexto clínico, social, e econômico, tanto para os doentes quanto para os prestadores de serviços em saúde pública [9]. Portanto, promover uma melhoria na prática gerencial e assistencial dos cuidados de saúde de enfermagem na área de nefrologia, de forma a garantir uma maior adesão terapêutica do doente renal crônico frente a necessidade da HD.

Este estudo teve como objetivo avaliar o perfil sociodemográfico, clínico e a adesão a HD dos pacientes renais crônicos em uma clínica de referência.

\section{Material e métodos}

Trata-se de um estudo descritivo, com abordagem quantitativa. A pesquisa foi desenvolvida nos meses de outubro de 2017 até janeiro de 2018 em um centro de diálise localizado no município de Juazeiro, estado da Bahia, com 320 pacientes renais crônicos cadastrados. A clínica oferece o tratamento diário em três turnos, exceto aos domingos. A população alvo foram doentes renais crônicos que estavam em tratamento hemodialítico. Para a seleção dos sujeitos, utilizou-se o processo de amostragem aleatório simples. O cálculo amostral considerou a utilização de variáveis categóricas, intervalo de confiança de $95 \%$, erro amostral de $5 \%$ e prevalência estimada de 50\% perfazendo uma amostra de 175 indivíduos, considerando uma perda por recusa totalizando a amostra final de 174 participantes.

Os critérios de inclusão de participantes do estudo foram: estar em tratamento renal hemodialítico há pelo menos três meses, independentemente da idade, sexo e grau de instrução e que aceitaram participar da pesquisa após o sorteio e assinaram o Termo de Consentimento Livre Esclarecido (TCLE). Os critérios de exclusão foram pacientes que não estavam admitidos na clínica de estudo, não aceitaram participar da pesquisa e se negaram a assinar o TCLE.

Dentre as variáveis dependentes da pesquisa estão: idade, sexo, profissão, horário da sessão, estado civil, escolaridade, renda familiar, raça, religião, causa que levou o indivíduo ao tratamento, possuir um cuidador, exercer alguma atividade laboral. Além disso, foi avaliada a variável independente realização da HD.

Para a coleta de dados foi utilizado o Questionário de avaliação sobre a adesão do portador de doença renal crônica em hemodiálise (QA - DRC-HD) validado e adaptado no Brasil no ano de 2017 [10]. Ele contém 46 questões divididas por blocos em quatro domínios: realização da HD, regime hídrico, regime dietético, regime medicamentoso. Em seu domínio hemodiálise traz 14 perguntas dentre as quais três permitem avaliar como está a adesão ao tratamento hemodialítico. Essas perguntas são consideradas precursoras para avaliação da adesão, sendo respostas categorizadas com escores [10].

O estudo relaciona o fato de ser aderente ou não ao tratamento, consistindo em o paciente responder as seguintes perguntas: se faltou alguma vez durante o mês passado para realização da HD, quantas vezes foram solicitadas a redução do tempo programado para 0 tratamento, e qual foi o tempo de redução. Os dados sociodemográficos e causa da doença renal crônica foram coletados como informações complementares, a partir de um instrumento confeccionado pelas pesquisadoras. A construção do banco de dados foi por dupla digitação, para minimizar os erros de inserção das variáveis analisadas.

A análise dos dados se deu por meio da estatística descritiva considerando a distribuição de frequência em números absolutos e relativos para as variáveis categóricas e as medidas de tendência central e dispersão como média e desvio padrão para as variáveis numéricas. Adotouse Intervalo de Confiança de $95 \%$ para a média e para a proporção considerando a distribuição binomial, neste último. Foram utilizados os programas Microsoft Office Excel 2013 e Stata versão 14.0 para análise dos dados.

Esta pesquisa foi aprovada pelo Comitê de Ética em Pesquisa da Universidade de Pernambuco atendendo a resolução 466/12 do Conselho Nacional de Saúde, em 03 de outubro de 2017, sob parecer ํㅡ․311.915.

\section{Resultados}

Dos 174 participantes, $103(59,2 \%)$ eram do sexo masculino, a média de idade foi de 51,4 anos, $48(27,6 \%)$ eram agricultores, $71(40,8 \%)$ eram casados, e $103(59,2 \%)$ possuíam ensino fundamental incompleto. Quanto a renda familiar, $113(64,9 \%)$ possuíam até um salário mínimo. A religião católica teve uma prevalência maior 103 (59,2\%) dentre as outras religiões. 
Tabela I - Dados sociodemográficos de pacientes renais crônicos em um centro de hemodiálise, Juazeiro/BA, Brasil, out/2017-jan/2018.

\begin{tabular}{|c|c|c|c|c|}
\hline \multirow{2}{*}{ Idade } & \multirow{2}{*}{$\begin{array}{l}\text { Média } \\
51,4 \\
\end{array}$} & \multirow{2}{*}{$\begin{array}{l}\text { DP } \\
14,9 \\
\end{array}$} & \multicolumn{2}{|c|}{ IC $95 \%{ }^{\dagger}$} \\
\hline & & & 49,1 & 53,6 \\
\hline & n & $\%$ & \multicolumn{2}{|c|}{ IC $95 \% \neq$} \\
\hline \multicolumn{5}{|l|}{ Sexo } \\
\hline Feminino & 71 & 40,8 & 33,7 & 48,3 \\
\hline Masculino & 103 & 59,2 & 51,7 & 66,3 \\
\hline \multicolumn{5}{|l|}{ Profissão } \\
\hline Outros & 41 & 23,6 & 17,8 & 30,5 \\
\hline Agricultor & 48 & 27,6 & 21,4 & 34,8 \\
\hline Sem profissão & 24 & 13,8 & 9,4 & 19,8 \\
\hline Aposentado & 36 & 20,7 & 15,3 & 27,4 \\
\hline Motorista & 8 & 4,6 & 2,3 & 9,0 \\
\hline Auxiliar de serviços gerais & 6 & 3,45 & 1,5 & 7,5 \\
\hline Doméstica & 6 & 3,45 & 1,5 & 7,5 \\
\hline Funcionário público & 5 & 2,87 & 1,2 & 6,8 \\
\hline \multicolumn{5}{|l|}{ Estado civil } \\
\hline Casado & 71 & 40,8 & 33,7 & 48,3 \\
\hline Solteiro & 45 & 25,9 & 19,8 & 33,0 \\
\hline Divorciado & 16 & 9,2 & 5,7 & 14,5 \\
\hline Viứvo & 12 & 6,9 & 3,9 & 11,8 \\
\hline União estável & 30 & 17,2 & 12,3 & 23,7 \\
\hline \multicolumn{5}{|l|}{ Escolaridade } \\
\hline Ensino fundamental incompleto & 103 & 59,2 & 51,7 & 66,3 \\
\hline Ensino fundamental completo & 21 & 12,1 & 8,0 & 17,9 \\
\hline Ensino médio incompleto & 12 & 6,9 & 3,9 & 11,8 \\
\hline Ensino médio completo & 32 & 18,4 & 13,3 & 24,9 \\
\hline Ensino superior & 6 & 3,5 & 1,5 & 7,5 \\
\hline \multicolumn{5}{|l|}{ Rend a familiar } \\
\hline Até um salário mínimo & 113 & 64,9 & 57,5 & 71,7 \\
\hline Entre 1 e 3 salários mínimos & 52 & 29,9 & 23,5 & 37,2 \\
\hline Entre 3 e 5 salários mínimos & 7 & 4,0 & 1,9 & 8,3 \\
\hline Acima de 5 salários mínimos & 2 & 1,2 & 0,3 & 4,5 \\
\hline \multicolumn{5}{|l|}{ Religião } \\
\hline Católica & 103 & 59,2 & 51,7 & 66,3 \\
\hline Evangélica & 39 & 22,4 & 16,8 & 29,3 \\
\hline Espirita & 26 & 14,9 & 10,3 & 21,1 \\
\hline Outras & 6 & 3,5 & 1,5 & 7,5 \\
\hline
\end{tabular}

†IC95\% - Intervalo de Confiança de 95\% para a média; †IC95\% - Intervalo de Confiança de 95\% para a proporção assumindo a distribuição binomial.

Dentre as patologias registradas, a HAS esteve presente em $61(35,1 \%)$ pacientes, a média de anos dos pacientes em HD foi de 4,6 anos. Apenas três $(1,7 \%)$ dos pacientes já fizeram diálise peritoneal anterior a HD. O tipo de locomoção mais utilizado pelos entrevistados foi o transporte da prefeitura de sua morada, e em sua maioria compareceram sozinhos ao centro de diálise. Em relação ao número de sessões, predominou a periodicidade de sessões de três vezes por semana, durante 4 horas, e a maioria dialisava no segundo turno. Considerando os dias e horários da realização da HD, 145 (83,3\%) responderam que estão convenientes.

Sobre a adesão terapêutica do doente renal crônico ao tratamento de HD, foram encontrados $154(88,5 \%)$ pacientes que aderiram. Entre os pacientes que relataram faltar a HD, a principal razão foi problemas com transporte representando $(4,0 \%)$. Em relação a razão da falta à terapia, $154(88,5 \%)$ não faltaram as sessões de HD. Com relação ao fato de não querer ir ou não poder ir, um (25\%) justificou se sentir mal fisicamente. $145(83,3 \%)$ dos participantes da pesquisa não solicitaram a redução do tempo programado para a terapia dialítica. 
Tabela II - Características clínicas e perfil de tratamento de pacientes renais crônicos submetidos a HD, Juazeiro/BA, Brasil, out/2017-jan/2018.

\begin{tabular}{|c|c|c|c|c|}
\hline & Média & DP & IC $95 \%{ }^{\dagger}$ & \\
\hline Tempo de HD em anos & 4,6 & 4,0 & 4,0 & 5,3 \\
\hline & $\mathrm{N}$ & $\%$ & IC $95 \% \neq$ & \\
\hline \multicolumn{5}{|l|}{ Patologias } \\
\hline Outras & 31 & 17,8 & 12,8 & 24,3 \\
\hline Diabetes mellitus & 35 & 20,1 & 14,8 & 26,8 \\
\hline Hipertensão arterial & 61 & 35,1 & 28,3 & 42,5 \\
\hline Lupus & 3 & 1,7 & 0,6 & 5,3 \\
\hline Glomerulopatias & 13 & 7,5 & 4,4 & 12,5 \\
\hline Indeterminada & 31 & 17,8 & 12,8 & 24,3 \\
\hline \multicolumn{5}{|l|}{ Dialise peritoneal } \\
\hline Não & 171 & 98,3 & 94,7 & 99,4 \\
\hline Sim & 3 & 1,7 & 0,6 & 5,3 \\
\hline \multicolumn{5}{|l|}{ Transporte utilizado } \\
\hline Carro & 33 & 19,0 & 13,8 & 25,6 \\
\hline Önibus & 6 & 3,5 & 1,5 & 7,5 \\
\hline Táxi & 5 & 2,9 & 1,2 & 6,8 \\
\hline Transporte da pref eitura & 61 & 35,1 & 28,3 & 42,5 \\
\hline Outros & 69 & 39,7 & 32,6 & 47,2 \\
\hline \multicolumn{5}{|l|}{ Acompan hante } \\
\hline Venho sozinho & 114 & 65,5 & 58,1 & 72,3 \\
\hline Meus pais (pai e mãe) & 3 & 1,7 & 0,6 & 5,3 \\
\hline Companheiro ( marido e esposa) & 26 & 14,9 & 10,3 & 21,1 \\
\hline Filho & 19 & 10,9 & 7,0 & 16,6 \\
\hline Amigo & 2 & 1,2 & 0,3 & 4,5 \\
\hline Outros & 10 & 5,8 & 3,1 & 10,4 \\
\hline \multicolumn{5}{|l|}{$\mathrm{N}^{\circ}$ de sessões da HD } \\
\hline 3 vezes & 173 & 99,4 & 96,0 & 99,9 \\
\hline 4 vezes ou mais & 1 & 0,6 & 0,1 & 4,0 \\
\hline \multicolumn{5}{|l|}{ Tempo de duração HD } \\
\hline Menos de três horas & 1 & 0,6 & 0,1 & 4,0 \\
\hline Três horas & 15 & 8,6 & 5,2 & 13,9 \\
\hline Três horas e 30 min & 16 & 9,2 & 5,7 & 14,5 \\
\hline Quatro horas & 142 & 81,6 & 75,1 & 86,7 \\
\hline \multicolumn{5}{|l|}{ Turno da HD } \\
\hline $1^{\circ}$ Turno & 49 & 28,2 & 21,9 & 35,4 \\
\hline $2^{\circ}$ Turno & 62 & 35,6 & 28,8 & 43,1 \\
\hline $3^{\circ}$ Turno & 48 & 27,6 & 21,4 & 34,8 \\
\hline $4^{\circ}$ Turno & 15 & 8,6 & 5,2 & 13,9 \\
\hline \multicolumn{5}{|l|}{ Dias/ ho rários convenientes } \\
\hline Sim & 145 & 83,3 & 77,0 & 88,2 \\
\hline Não, comparecer ce do ao œentro de HD & 6 & 3,5 & 1,5 & 7,5 \\
\hline Não, comparecer ao centro de HD tarde & 20 & 11,5 & 7,5 & 17,2 \\
\hline Outros & 3 & 1,7 & 0,6 & 5.3 \\
\hline
\end{tabular}

†IC95\% - Intervalo de Confiança de 95\% para a média; łIC95\% - Intervalo de Confiança de $95 \%$ para a proporção assumindo a distribuição binomial.

Tabela III - Adesão do doente renal crônico ao tratamento de hemodiálise em um centro de referência, Juazeiro/BA, Brasil, 2017-2018.

\begin{tabular}{|c|c|c|c|c|}
\hline \multirow{3}{*}{$\begin{array}{l}\text { Variável } \\
\text { Adesão ao tratamen to de HD } \\
\text { Não aderente }\end{array}$} & \multirow[t]{2}{*}{$\mathbf{N}$} & \multirow[t]{2}{*}{$\%$} & \multicolumn{2}{|c|}{ IC $95 \%$} \\
\hline & & & & \\
\hline & 20 & 11,5 & 7,5 & 17,2 \\
\hline Aderente & 154 & 88,5 & 82,8 & 92,5 \\
\hline \multicolumn{5}{|l|}{ Razão da falta } \\
\hline Não faltei & 154 & 88,5 & 82,8 & 92,5 \\
\hline Problemas com transporte & 7 & 4,0 & 1,9 & 8,3 \\
\hline Resolução de problemas particulares & 1 & 0,6 & 0,1 & 4,0 \\
\hline Atendimento médico & 3 & 1,7 & 0,6 & 5,3 \\
\hline Atendimento de emergência & 1 & 0,6 & 0,1 & 4,0 \\
\hline Não queria ir & 4 & 2,3 & 0,9 & 6,0 \\
\hline Outros & 4 & 2,3 & 0,9 & 6,0 \\
\hline \multicolumn{5}{|l|}{ Justificativa para não querer ou não poder ir } \\
\hline Porque estava me sentindo mal fisicamente & 1 & 25,0 & 0,5 & 95,9 \\
\hline Outros & 3 & 75,0 & 4,1 & 99,5 \\
\hline \multicolumn{5}{|c|}{ Quantas vezes o paciente solicitou redução do tempo da HD no mês } \\
\hline passad o & & & & \\
\hline Não se aplica: não reduziu o tempo & 145 & 83,3 & 77,0 & 88,2 \\
\hline Redução do tempo uma ou mais vezes & 29 & 16,7 & 11,8 & 23,0 \\
\hline
\end{tabular}


Com relação à orientação dos profissionais sobre o tratamento dialítico, percebeu-se que $30(17,2 \%)$ responderam que os cuidadores falaram toda semana sobre a importância de não faltar à HD. Já em relação à orientação do profissional sobre a importância de realizar o tratamento sem diminuir o tempo, $50(28,7 \%)$ responderam que o profissional de saúde fala sobre a importância de fazer a hemodiálise sem diminuir o tempo programado.

Tabela IV - Orientações profissionais sobre o tratamento dialítico em um centro de referência, Juazeiro/BA, Brasil, 2017-2018.

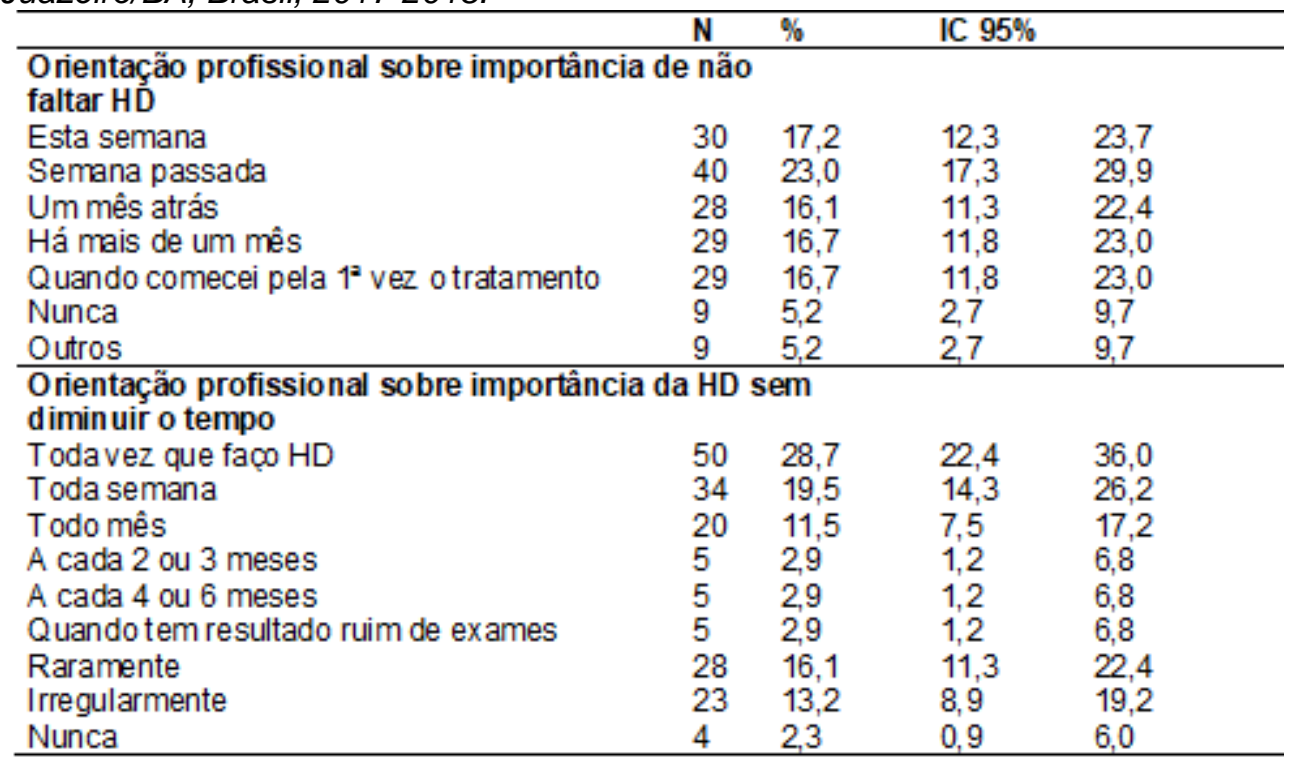

Sobre a percepção dos pacientes quanto à importância de cumprir as sessões de HD, $131(75,3 \%)$, responderam que acham extremamente importante cumprir a HD. A justificativa mais prevalente sobre o porquê é importante cumprir a programação, $85(48,9 \%)$ foi que entendem que seus rins não funcionam adequadamente e, por isso, precisam cumprir a programação. Considerando a dificuldade em permanecer durante toda sessão de HD, 131 $(75,3 \%)$ responderam não possuir nenhuma dificuldade.

Tabela V - Percepção dos pacientes sobre a importância de cumprir as sessões de hemodiálise em um centro de referência, Juazeiro/BA, Brasil, 2017-2018.

\begin{tabular}{|c|c|c|c|c|}
\hline \multicolumn{2}{|c|}{ Percepção do paciente sobre quão importante cumprir HD } & & \multicolumn{2}{|c|}{ IC $95 \%$} \\
\hline Extremamente importante & 34 & 19,5 & 14,3 & 26.2 \\
\hline Muito importante & 131 & 75,3 & 68,3 & 81,2 \\
\hline Moderadamente importante & 4 & 2,3 & 0,9 & 6,0 \\
\hline Não é importante & 5 & 2,9 & 1,2 & 6,8 \\
\hline \multicolumn{5}{|l|}{ Porque é importante a HD } \\
\hline \multicolumn{4}{|l|}{ Poraue entende que os rins não funcionam } & 56,3 \\
\hline Porque cumprir a programação é importante para & & & & \\
\hline $\begin{array}{l}\text { manter o corpo saudável } \\
\text { Porque o profissional me disse para cumprir a }\end{array}$ & 75 & 43,1 & 35,9 & 50,6 \\
\hline programação & 3 & 1,7 & 0,6 & 5,3 \\
\hline Porque já fiquei doente depois de faltar HD & 3 & 1,7 & 0,6 & 5,3 \\
\hline Porque eu já fui hospitalizado depois de faltar HD & 3 & 1,7 & 0,6 & 5,3 \\
\hline Eu não acho importante cumprir HD & 3 & 1,7 & 0,6 & 5,3 \\
\hline Outros & & 1,2 & 0,3 & 4,5 \\
\hline \multicolumn{5}{|c|}{$\begin{array}{l}\text { Quanta dificuldade em permanecer durante toda sessão de } \\
\text { HD }\end{array}$} \\
\hline Nenhuma dificuldade & 131 & 75,3 & 68,3 & 81,2 \\
\hline Um pouco de dificuldade & 21 & 12,1 & 8,0 & 17,9 \\
\hline Moderada dificuldade & 8 & 4,6 & 2,3 & 9,0 \\
\hline Muita dificuldade & 10 & 5,8 & 3,1 & 10,4 \\
\hline Extrema dificuldade & 4 & 2,3 & 0,9 & 6,0 \\
\hline
\end{tabular}


Dentre os dados obtidos no estudo houve a prevalência maior de pacientes do sexo masculino, ensino fundamental incompleto. Um estudo realizado em São Luiz, Maranhão, Brasil, cujo objetivo era identificar fatores socioeconômicos, demográficos, clínicos nutricionais e laboratoriais de pacientes em terapia renal substitutiva revelaram 0 mesmo perfil sociodemográfico [6]. A profissão de agricultor mostrou-se com um número relevante dentre os participantes, e a religião católica como opção de escolha.

O perfil epidemiológico do renal crônico encontrado em outro estudo corroboram os dados encontrados nesta pesquisa em que a doença renal crônica aparece predominantemente no sexo masculino [11]. Fato esse que pode estar relacionado a vulnerabilidade dos homens as doenças do aparelho circulatório dentre elas a HAS. A Política Nacional de Integração a Saúde do Homem revela que os homens evitam contatos com consultórios médicos, corredores de unidades de saúde, tornando-os avessos a prevenção e autocuidado, protelando a procura do atendimento médico [12].

A HAS seguida pelo diabetes mellitus foram as morbidades que mais acometeram os indivíduos deste estudo. Um estudo semelhante descreve aproximação com os dados encontrados revelando a hipertensão seguida do diabetes mellitus como principal causa que leva a insuficiência renal [4]. Dados do Inquérito Brasileiro de Diálise realizado no ano de 2016, publicado pela Sociedade Brasileira de Nefrologia (SBN), também mostram como casos mais frequentes para o diagnóstico de DRC, a hipertensão arterial (34\%) e o diabetes (30\%) [2].

A clínica em estudo atende pacientes de municípios circunvizinhos da Bahia, e estados como Pernambuco e Piauí, tornando-se necessário a utilização do transporte da prefeitura para o deslocamento até o centro de HD. Diante disso, a pesquisa mostrou que um número considerável de usuários faz uso desse tipo de transporte para ir as sessões, a maioria comparece ao serviço sozinho, e possui renda de até um salário mínimo.

A dependência do uso do transporte foi considerada uma causa de faltas e atrasos nas sessões de HD, tal resultado pode estar relacionado as dificuldades financeiras relatadas, tornando-se um agravante custear o translado até o centro [13]. Considerando os efeitos da DRC e seus impactos, é apontado dentre tantas restrições, a dificuldade em retornar sua atividade laboral por limitações físicas e psíquicas [14].

Pacientes em terapia renal substitutiva realizam sessões de (HD) três vezes por semana, durante quatro horas. Esse tratamento utiliza equipamentos de alta tecnologia para realização de depuração do sangue para que ocorra uma remoção adequada das toxinas [15]. O presente estudo demostrou que os pacientes da clínica responderam que os horários programados das sessões de HD estavam convenientes, tornando-se um dado de grande relevância para uma maior adesão ao tratamento. Uma pesquisa demonstrou que quanto maior a idade do indivíduo menor é o número de faltas na HD, consequentemente melhor é a adesão, a média de idade de seus participantes foi de 54,7 anos [14]. Dado aproximado com o deste estudo, na qual a média de idade foi de 51,4 anos.

Quando se refere ao impacto que o tratamento dialítico causa ao paciente, estes estão relacionados às repercussões psicossociais e eventos negativos, podendo interferir em sua qualidade de vida [11]. Durante as sessões de HD, podem ocorrer uma série de eventos estressores fisiológicos que incluem a hipotensão arterial, náuseas, cefaleia, arritmias, fadiga, dor, hematomas, sangramentos no local das punções da fístula arteriovenosa, entre outros [16]. Esses fatores podem tanto influenciar na falta desses indivíduos quanto na solicitação de redução do tempo da terapia.

Os participantes da pesquisa demonstraram ser bem aderentes, uma vez que a maioria não solicitou a redução do tempo da HD, compareceram com frequência para realização do tratamento, e responderam não terem faltado às sessões. Aos que responderam ter faltado alguma vez, a principal razão de falta estava em não querer ir, ou não poder ir, sendo justificado porque estava se sentindo mal fisicamente.

No que se refere as orientações feitas pelos profissionais de saúde, foi percebido que a maioria fala sobre a importância de não faltar sessões de HD pelo menos semanalmente. Além disso, a maioria dos profissionais também faz orientações acerca da não redução do tempo programado da HD toda vez que o paciente comparece ao serviço para o tratamento. Contrapondo com estes achados, uma pesquisa que avaliou o domínio HD mostrou em seus resultados que a orientação pelos profissionais ocorria apenas quando o tratamento era iniciado pela primeira vez, dessa forma a não adesão esteve presente em 32\% dos usuários [17]. 
Os profissionais de saúde devem ser vigilantes quanto às questões relacionadas à adesão, identificando as inquietações e preocupações dos pacientes [18]. O enfermeiro nefrologista desempenha um papel crítico e valioso na qualidade dos cuidados, sendo uma prioridade garantir a alta qualidade na assistência [19]. Entre as principais intervenções de enfermagem a esses pacientes está a prevenção de infecções, promoção do autocuidado, orientações a família e ao paciente, dentre outras [20].

Quanto a percepção dos pacientes sobre a importância da realização do tratamento, eles entendiam que os rins funcionavam de forma inadequada e precisavam cumprir a programação, e não tinham dificuldade quanto a esse cumprimento. Além disso, foi observado que as orientações profissionais fornecidas tiveram grande relevância, fazendo com que a maioria dos pacientes compreendesse a importância de seu tratamento, repercutindo em uma boa adesão.

Como a clínica estudada possui certificação pela Organização Nacional Acreditação (ONA), nível dois plena, está atualmente em processo para adquirir o nível três, considerado padrão ouro. Deste último nível no processo de acreditação hospitalar, demonstra a busca constante na prestação de um serviço de qualidade. Os dados aqui apresentados reforçam essa questão, uma vez que os pacientes percebiam nos profissionais comprometimento com a assistência. O incentivo para alcançar a qualidade da assistência vem do processo de acreditação hospitalar pressupondo medição de desempenho e ações de educação [21]. Esta é uma ferramenta que os serviços de saúde utilizam para alcançar a qualidade da assistência [22].

Algumas limitações do estudo estiveram relacionadas ao nível de escolaridade baixo dos participantes e a longa extensão do questionário. Esses fatores resultaram na dificuldade de entendimento de algumas perguntas, exigindo várias releituras, tornando-se cansativo tanto para as pesquisadoras quanto para os pacientes.

\section{Conclusão}

A pesquisa revelou que a maioria dos pacientes são homens, casados, agricultores, possuem baixa escolaridade, e religião prevalentemente católica. Aponta como principais fatores causais nos indivíduos em terapia hemodialítica, a hipertensão arterial e o diabetes mellitus. A maioria dos participantes se mostrou aderente à $\mathrm{HD}$, o que pode estar relacionado a qualidade do serviço prestado, por se tratar de uma clínica acreditada, que promove uma constante capacitação profissional. Percebeu-se ainda que existe o comprometimento por parte dos profissionais de saúde sobre o tratamento dialítico.

O estudo demonstrou ainda que os pacientes possuem uma boa percepção quanto a importância de cumprir as sessões de HD, além de considerarem importante o cumprimento dessa programação, bem como afirmarem não possuir dificuldades em permanecer nas sessões.

Avaliar a adesão do doente renal crônico ao tratamento hemodialítico constitui-se uma ferramenta a ser utilizada na prática gerencial e assistencial do serviço de nefrologia. Ponderar outros domínios de análise como adesão aos medicamentos, ingesta de líquidos e dieta, podem ampliar essa ferramenta e possibilitar incremento para a qualidade de vida destas pessoas.

\section{Referências}

1. Stum EMF, Kirchner RM, Guido LA, Benetti ERR, Belasco AGS, Sesso RCC et al. Intervenção educacional de enfermagem para redução da hiperfosfatemia em pacientes em hemodiálise. Rev Bras Enferm 2017;70(1):31-8. https://doi.org/10.1590/0034-7167-2016-0015

2. Sesso RC, Lopes AA, Thomé FS, Lugon JR, Martins CT. Brazilian Chronic Dialysis Survey 2016. J Bras Nefrol 2017;39(3):261-66. https://doi.org/10.5935/01012800.20170049

3. Cristovão AFAJ. Eficácia das restrições hídrica e dietética em pacientes renais crônicos em hemodiálise. Rev Bras Enferm 2015;68(6):1154-62. https://doi.org/10.1590/00347167.2015680622i

4. Teixeira FIR, Lopes ML, Silva GAS, Santos RF. Sobrevida de pacientes em hemodiálise em um hospital universitário. J Bras Nefrol 2015;37(1):64-71. https://doi.org/10.5935/0101-2800.20150010

5. Guedes KD, Guedes HM. Qualidade de vida do paciente portador de insuficiência renal crônica. Rev Ciência e Saúde 2012;5(1):48-53. https://doi.org/10.15448/1983$\underline{652 X .2012 .1 .9734}$ 
6. Cavalcante MCV, Lamy ZC, Lamy Filho F, França AKTC, Santos AM, Thomaz EBA, Fonseca et al . Fatores associados à qualidade de vida de adultos em hemodiálise em uma cidade do nordeste do Brasil. J Bras Nefrol 2013;35(2):79-86.

https://doi.org/10.5935/0101-2800.20130014

7. Silva RAR, Souza VL, Oliveira GJN, Silva BCO, Rocha CCT, Holanda JRR. Estratégias de enfrentamento utilizadas por pacientes renais crônicos em tratamento hemodialítico. Esc Anna Nery 2016;20(1):147-54. https://doi.org/10.5935/1414-8145.20160020

8. Martins RR, Alchierri JC. Avaliação psicológica na aderência terapêutica de tratamento hemodialítico. Psicol Argumento 2013;31(72):127-36. https://doi.org/10.7213/psicol.argum.7595

9. Pinheiro J. Autonomia e aderência na pessoa com doença renal crônica. Rev Bioética 2011;19(1):219-29.

10. Lins SMSB, Leite JL, Godoy S, Fuly PSC, Araújo STC, Silva IR. Validação do questionário de adesão do paciente renal crônico brasileiro em hemodiálise. Rev Bras Enferm 2017;70(3):558-65. https://doi.org/10.1590/0034-7167-2016-0437

11. Oliveira CS, Silva EC, Ferreira LW, Skalinski LM. Perfil dos pacientes renais crônicos em tratamento hemodialítico. Rev Baiana Enferm 2015;29(1):42-9. Disponível: http://www.portalseer.ufba.br/index.php/enfermagem/article/view/12633/9541

12. Martins AM, Malamut BS. Análise do discurso da Política Nacional de Atenção Integral à Saúde do Homem. Saude Soc 2013;22(2):429-40. https://doi.org/10.1590/S010412902013000200014

13. Maciel CG, Ferraz RN, França VV, Frazão ISAK de OTB. Adesão ao tratamento hemodialítico: percepção dos pacientes renais crônicos. Cogitare Enferm 2015;20(3):540-7.

14. Oliveira APB, Schmidt DB, Amatneeks TM, Santos JC dos, Cavallet LHR, Michel RB. Quality of life in hemodialysis patients and the relationship with mortality, hospitalizations and poor treatment adherence. J Bras Nefrol 2016;38(4):411-20. https://doi.org/10.5935/0101-2800.20160066

15. Noleto LC, Fonseca AC, Luz MHBA, Batista OMA, Pereira AFM. O papel dos profissionais de enfermagem no cuidado ao paciente em tratamento hemodialítico?: revisão integrativa. Rev Enferm UFPE Line 2015;9:1580-6. https://doi.org/10.5205/reuol.8463-73861-2-SM.0910sup201526

16. Beuter M, Muller LR, Brondani CM, Pauletto MR, Timm MB, Marilene $N$ et al. A adesão de individuos em terapia hemodiálitica. Rev Pesqui Cuid Fundam Online 2013;5(2):3558-66.

17. Lins SMSB, Leite JL, Godoy S, Tavares JMAB, Rocha RG, Silva FVC. Adesão de portadores de doença renal crônica em hemodiálise ao tratamento estabelecido. Acta Paul Enferm 2018;31(1):54-60. https://doi.org/10.1590/1982-0194201800009

18. Ghimire S, Castelino RL, Jose MD, Zaidi STR. Medication adherence perspectives in haemodialysis patients?: a qualitative study. Nephrol 2017;18(1):167. https://doi.org/10.1186/s12882-017-0583-9

19. Nobahar M. Exploring experiences of the quality of nursing care among patients, nurses, caregivers and physicians in a haemodialysis department. J Ren Care 2017;43(1):50-9. https://doi.org/10.1111/jorc. 12187

20. Frazão CMFQ, Delgado MF, Araújo MGA, Silva FBB, Sá JD, Lira ALBC et al. Cuidados de enfermagem ao paciente renal crônico em hemodiálise. Rev Rene 2014;15(4):7019.

21. Mendes GHS ,Mirandola TBS. Acreditação hospitalar como estratégia de melhoria?: impactos em seis hospitais acreditados. Gest Prod 2015;22(3):636-48. https://doi.org/10.1590/0104-530X1226-14

22. Oliveira JLC, Matsuda LM. Vantagens e dificuldades da acreditação hospitalar: A voz dos gestores da qualidade. Esc Anna Nery 2016;20(1):63-9. https://doi.org/10.5935/1414-8145.20160009 